\title{
Impact of alpha 1-antitrypsin deficiency and prior augmentation therapy on patients' survival after lung transplantation
}

To the Editor:

Alpha 1-antitrypsin (AAT) deficiency (AATD) is a genetic condition characterised by low serum levels of AAT and is associated with a high risk of developing early-onset pulmonary emphysema, especially in cigarette smokers [1]. The most common deficiency alleles among Caucasians are $\mathrm{Pi}^{\star} \mathrm{Z}$ (Glu342Lys) and $\mathrm{Pi}^{\star} \mathrm{S}$ (Glu264Val), and the majority of individuals with severe AATD are Pi type ZZ. According to available literature, PiZZ AATD comprises about 1-5\% of all chronic obstructive pulmonary disease (COPD) cases [2]. Given the importance of the protease/anti-protease imbalance in causing $\mathrm{Pi}^{\star} \mathrm{Z}$-related emphysema [3], intravenous infusions of exogenous AAT are used as a specific augmentation therapy. Data from large interventional and observational studies suggest that decline in lung function and overall mortality decelerates in augmented compared to non-augmented patients [4]. Despite this therapy, it is impossible to halt the progression of emphysema in many patients and for those who develop end-stage lung disease, lung transplantation (LTx) remains the only option [5]. AATD is the fourth-most common reason for LTx, accounting for about $6 \%$ of all adult lung transplants [6]; however, there are only a few studies of post-LTx outcomes of patients with AATD [7, 8]. Evidence for the use of augmentation therapy after LTx is insufficient, and there is no consensus whether LTx recipients with AATD should receive augmentation therapy. According to the data, only 13-19\% of AATD patients receive augmentation therapy after LTx [7]. Furthermore, the putative influence of previous augmentation therapy on lung recipients who discontinued this therapy following transplantation is entirely unknown.

To determine the 10-year survival rates for the AATD recipients who have had or have not had augmentation therapy prior to LTx, we conducted a retrospective review of a large transplantation database at the Clinic for Pneumology, Hannover Medical School, Germany. The Ethics Committee of Hannover Medical School approved the study. Interestingly, Hannover's transplant centre does not provide augmentation therapy to post-LTx patients with AATD. Hannover Medical School is one of the world's largest and leading lung transplant centres routinely performing LTx in patients with end-stage lung disease related to COPD, AATD, cystic fibrosis, sarcoidosis and other complex conditions. Most patients with these diagnoses were transferred from other centres; therefore, our centre has no influence regarding best patient care and augmentation therapy before LTx. We have no information whether augmentation was offered for AATD patients with more severe and earlier disease, in the presence of coexisting liver disease, etc. We confirm that all patients with end-stage lung diseases fulfilled the criteria for LTx. Data were analysed with IBM SPSS Statistics 24.0 (IBM Corp, Armonk, NY, USA) and STATA 13.0 (State Corp LP, College Station, TX, USA) software. Kaplan-Meier statistics were used to estimate survival rates with statistical significance assessed by log rank test.

Between October 1999 and December 2012, a total of 1020 patients underwent LTx including 351 patients with COPD/emphysema, of which 29.9\% $(n=105)$ had inherited AATD. For analysis, only patients with complete documentation of demographic data were included. We matched 246 non-AATD with COPD/ emphysema as a primary diagnosis (hereafter referred to as COPD) and 105 AATD-COPD/emphysema patients (91 PiZZ and 14 PiSZ, hereafter referred to as AATD). Before LTx, 58 (55\%) of AATD patients

@ERSpublications

AATD patients who received augmentation therapy prior to LTx show worse survival rates http://ow.ly/tfgX30e5cVW

Cite this article as: Conrad A, Janciauskiene S, Köhnlein T, et al. Impact of alpha 1-antitrypsin deficiency and prior augmentation therapy on patients' survival after lung transplantation. Eur Respir J 2017; 50: 1700962 [https://doi.org/10.1183/13993003.00962-2017]. 
(52 PiZZ and 6 PiSZ) received augmentation therapy (AATD ${ }^{\text {aug }+}$ ). Out of 351 LTx patients, 92.3\% were bilateral lung recipients: $90.7 \%$ COPD, $94.8 \%$ AATD $^{\text {aug }+}$ and $97.9 \%$ AATD $^{\text {aug- }}$. There was no significant difference between $\mathrm{AATD}^{\text {aug+ }}$ and $\mathrm{AATD}^{\text {aug- }}$ groups in terms of mean \pm SD age at the time of LTx: 51.5 \pm 6.7 years, $n=58$ and $49.1 \pm 6.7$ years, $n=47, p=0.075$. However, COPD patients were older $(54.0 \pm 7.2$ years, $\mathrm{n}=246)$ than $\mathrm{AATD}^{\text {aug }+}(\mathrm{p}=0.018)$ or $\mathrm{AATD}^{\text {aug- }}(\mathrm{p}<0.001) .45 \%$ of COPD, $67 \%$ of AATD ${ }^{\text {aug }}$ and $64 \%$ of AATD $^{\text {aug- }}$ LTx patients were male.

10 years after LTx, 173 out of 351 (49.3\%) patients developed chronic lung allograft dysfunction (CLAD) and $182(51.9 \%)$ died. Surprisingly, the AATD ${ }^{\text {aug+ }}$ group had the lowest overall post-LTx survival rate. As illustrated in figure 1a, the 10 -year survival rate within the $\mathrm{AATD}^{\text {aug+ }}$ group was only $26 \%$ compared to 69\% $(\mathrm{p}<0.001)$ for the AATD ${ }^{\text {aug- }}$ and 39\% $(\mathrm{p}=0.009)$ for the COPD groups. After 1 year, the survival rates were already significantly lower for the AATD $^{\text {aug+ }}$ (71\%) versus COPD (88\%) whereas after 3 years, the survival rates were significantly lower for the AATD $^{\text {aug+ }}(55 \%)$ versus AATD $^{\text {aug- }}$ (79\%) and COPD (74\%) groups (figure 1a).

An increased risk of death at each stage after LTx is associated with the development and progression of CLAD. However, our attempt to categorise the causes of death gave no explanation for the survival difference between the groups (figure 1b). Overall, the AATD ${ }^{\text {aug- }}$ group showed the best post-LTx survival, which is in line with survival rates recently presented for AATD LTx at Skåne University Hospital in Lund, Sweden [8]. The incidence of rejection is lower for younger LTx recipients [9] and thus the younger age at LTx of the AATD ${ }^{\text {aug- }}$ group probably favoured survival. Despite the facts that age at LTx did not differ between $\mathrm{AATD}^{\text {aug+ }}$ and $\mathrm{AATD}^{\text {aug- }}$ patients and that AATD ${ }^{\text {aug+ }}$ patients were younger than COPD patients, they nevertheless showed the lowest survival rates.

To address this conundrum, we speculated that the putative differences in the first-year outcomes between the studied groups might give us a hint as to why the AATD ${ }^{\text {aug }}$ group showed the worst post-LTx performance. We reviewed the clinical data of AATD patients (1999-2014) who attended the outpatient clinic for routine check-ups 1-year post-LTx at our institution. Of these patients, we found $47 \mathrm{AATD}^{\text {aug }}$ and $42 \mathrm{AATD}^{\text {aug-}}$, and we matched 40 COPD LTx patients. None of the three groups differed significantly with respect to baseline demographic data: age, gender, body mass index, previous history of smoking, dependency on a wheelchair, airway colonisation, lung function tests, pulmonary hypertension, oxygen therapy requirement, quality of life, frequency of exacerbation overall and with hospitalisations. The lung function tests and quality of life post-LTx improved comparably for all COPD and AATD patients. The

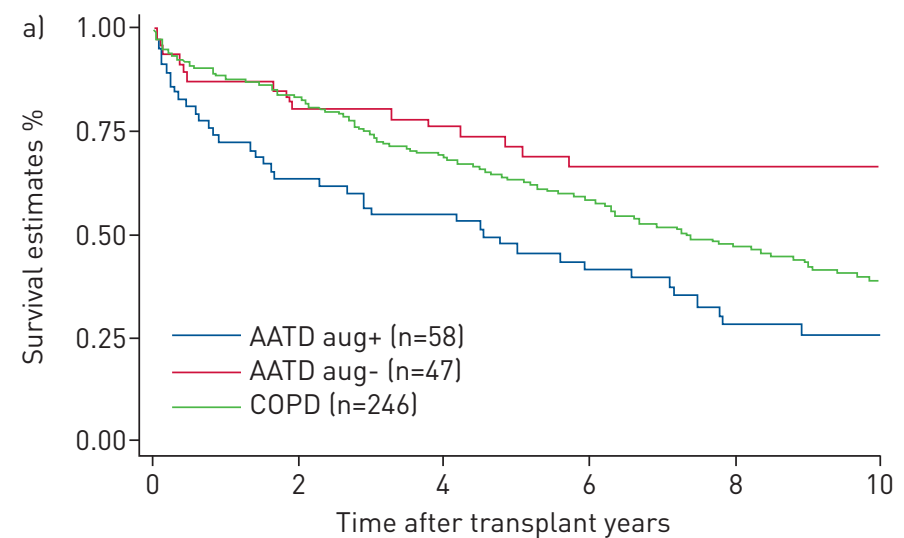

Percentage survival rates

\begin{tabular}{lcccccc}
\hline Years & $\begin{array}{c}\text { AATD aug } \\
\text { versus } \\
\text { AATD aug- }\end{array}$ & $p$-value & $\begin{array}{c}\text { AATD aug+ } \\
\text { versus } \\
\text { COPD }\end{array}$ & $p$-value & $\begin{array}{c}\text { AATD aug- } \\
\text { versus } \\
\text { COPD }\end{array}$ & $p$-value \\
\hline 1 & 71 versus 85 & 0.068 & 71 versus 88 & 0.001 & 85 versus 88 & 0.789 \\
3 & 55 versus 79 & 0.005 & 55 versus 74 & 0.002 & 79 versus 74 & 0.302 \\
5 & 46 versus 69 & 0.010 & 46 versus 63 & 0.004 & 69 versus 63 & 0.364 \\
10 & 26 versus 69 & $<0.001$ & 26 versus 39 & 0.009 & 69 versus 39 & 0.022 \\
\hline
\end{tabular}

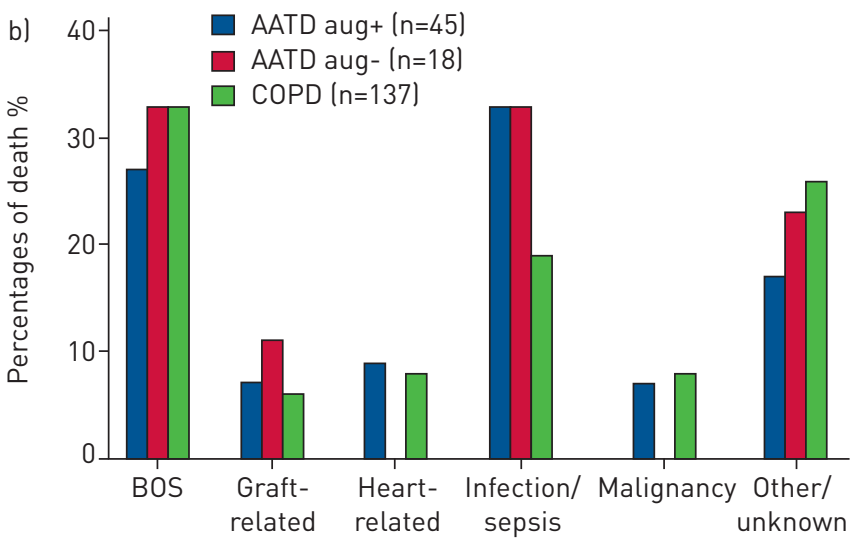

\begin{tabular}{lccc} 
Cause of death & AATD aug+ & AATD aug- & COPD \\
\hline BOS & $12(27 \%)$ & $6(33 \%)$ & $45(33 \%)$ \\
Graft-related & $3(7 \%)$ & $2(11 \%)$ & $8(6 \%)$ \\
Heart-related & $4(9 \%)$ & $0(0 \%)$ & $11(8 \%)$ \\
Infection/sepsis & $15(33 \%)$ & $6(33 \%)$ & $26(19 \%)$ \\
Malignancy & $3(7 \%)$ & $0(0 \%)$ & $11(8 \%)$ \\
Other/unknown & $8(18 \%)$ & $4(33 \%)$ & $36(26 \%)$
\end{tabular}

FIGURE 1 a) Kaplan-Meier survival analysis for lung transplants from 1999 to 2012, and b) comparisons of cause of death. AATD: alpha 1 -antitrypsin deficiency; aug+: received augmentation therapy; aug-: did not receive augmentation therapy; COPD: chronic obstructive pulmonary disease. 
occurrence of infections, rejections and airway complications remained very low and without significant differences among the groups. The comparison between $\mathrm{AATD}^{\text {aug+ }}$ and $\mathrm{AATD}^{\text {aug- }}{ }^{\text {revealed no difference }}$ in the number of days on respirator $(\mathrm{p}=0.66)$, days in intensive care unit $(\mathrm{p}=0.89)$, overall hospital stay $(p=0.14)$, rejection episodes $(p=0.09)$ and airway complications $(p=0.65)$. In the 6 -min walk distance (6MWD), the overall performance after LTx did not differ between COPD and AATD patients, independent of former augmentation therapy. However, COPD and AATD ${ }^{\text {aug- }}$ patients showed greater improvement in $\triangle 6 \mathrm{MWD}$ (6 min walk test slope after and prior-LTx) (median $+230 \mathrm{~m}, \mathrm{IQR}+170-312 \mathrm{~m}$ ) and (median $+212 \mathrm{~m}, \mathrm{IQR}+201-238 \mathrm{~m}$ ), respectively, relative to $\mathrm{AATD}^{\text {aug }}{ }^{\text {(median }}+186 \mathrm{~m}, \mathrm{IQR}+185-$ $207 \mathrm{~m}, \mathrm{p}=0.01$ ). $6 \mathrm{MWD}$ is significantly associated with post-transplant survival [10]. Therefore, although the lowest improvement in 6MWD alone among AATD ${ }^{\text {aug+ }}$ patients does not prove the lowest post-transplant survival, it still warrants further investigations. We speculate that long-term augmentation therapy may affect muscle adaptation for training, kidney function and lipid metabolism, among others, which play a critical role in patients' survival after LTx. For instance, after LTx, acute kidney injury often evolves into chronic kidney disease, which is a known cause of mortality [11]. Although all patients received nephrotoxic drugs after LTx, it is possible that $\mathrm{AATD}^{\text {aug }}{ }^{+}$patients are more sensitive. The effects of long-term augmentation therapy with AAT on kidney function are unknown. MEYER et al. [12] demonstrated that acute reperfusion injury and acute or chronic rejection increase AAT serum levels. Regrettably, AAT and creatinine levels from the early post-operative period were not available in our database. Secondly, AAT is considered to be an immunomodulatory therapy, which presumably compromises the immune system, and if discontinued after LTx, immunosuppressive therapy probably needs to be specifically adjusted for these AATD patients. In support of this assumption, other small studies have found that post-LTx survival rates of AATD patients with continued augmentation therapy did not differ from COPD [7].

The main conclusion with respect to AATD patient survival after LTx is that patients who received augmentation therapy prior to LTx show worse 10-year survival rates relative to AATD without prior augmentation as well as to those with general COPD. Currently, we are unable to provide an explanation for this unexpected finding. It could be that AATD patients who were receiving augmentation therapy prior to LTx have had more profound illness, and that could have impacted the post-transplant course. This topic warrants further prospective investigations. It is also possible that both prior augmentation therapy and/or therapy discontinuation have an adverse effect on post-LTx patient survival. Unfortunately, we could not address this question in a retrospective study. A prospective study design that includes post-LTx AATD patients continuing and discontinuing augmentation therapy will be required.

Anton Conrad ${ }^{1}$, Sabina Janciauskiene ${ }^{1,2,6}$, Thomas Köhnlein ${ }^{3}$, Jan Fuge ${ }^{1,2}$, Philipp Ivanyi ${ }^{4}$, Igor Tudorache ${ }^{2,5}$, Jens Gottlieb ${ }^{1,2}$, Tobias Welte ${ }^{1,2}$ and Thomas Fuehner ${ }^{1,2,6}$

${ }^{1}$ Dept of Respiratory Medicine, Hannover Medical School, Hannover, Germany. ${ }^{2}$ Biomedical Research in Endstage and Obstructive Lung Disease Hannover (BREATH), Member of the German Center for Lung Research (DZL), Hannover, Germany. ${ }^{3}$ Dept of Respiratory Medicine, Klinikum St. Georg gGmbH (Robert-Koch-Klinikum), Leipzig, Germany. ${ }^{4}$ Hematology, Hemostasis, Oncology and Stem Cell Transplantation, Hannover Medical School, Hannover, Germany. ${ }^{5}$ Dept of Cardiothoracic, Transplantation and Vascular Surgery, Hannover Medical School, Hannover, Germany. ${ }^{6}$ These authors contributed equally.

Correspondence: Thomas Fuehner, Dept of Respiratory Medicine, Hannover Medical School, 30623 Hannover, Germany. E-mail: fuehner.thomas@mh-hannover.de

Received: May 122017 | Accepted after revision: June 302017

Support statement: This study was funded by the German Center for Lung Research (DZL). Funding information for this article has been deposited with the Crossref Funder Registry.

Conflict of interest: Disclosures can be found alongside this article at erj.ersjournals.com

\section{References}

1 Silverman EK, Sandhaus RA. Clinical practice. Alpha1-antitrypsin deficiency. N Engl J Med. 2009; 360: 2749-2757.

2 Greulich T, Nell C, Hohmann D, et al. The prevalence of diagnosed $\alpha 1$-antitrypsin deficiency and its comorbidities: results from a large population-based database. Eur Respir J. 2017; 49: 1600154.

3 Janciauskiene S, Welte T. Well-known and less well-known functions of alpha-1 antitrypsin. Its role in chronic obstructive pulmonary disease and other disease developments. Ann Am Thorac Soc. 2016; 13: Suppl. 4, S280-S288.

4 Balbi B, Ferrarotti I, Miravitlles M. Efficacy of augmentation therapy for emphysema associated with $\alpha 1$-antitrypsin deficiency: enough is enough. Eur Respir J. 2016; 47: 35-38.

5 Teschler H. Long-term experience in the treatment of al-antitrypsin deficiency: 25 years of augmentation therapy Eur Respir Rev. 2015; 24: 46-51.

6 Yusen RD, Christie JD, Edwards LB, et al. The Registry of the International Society for Heart and Lung Transplantation: Thirtieth Adult Lung and Heart-Lung Transplant Report - 2013; focus theme: age. J Heart Lung Transplant. 2013; 32: 965-978. 
7 Banga A, Gildea T, Rajeswaran J, et al. The natural history of lung function after lung transplantation for alpha (1)-antitrypsin deficiency. Am J Respir Crit Care Med. 2014; 190: 274-281.

8 Fakhro M, Ingemansson R, Skog I, et al. 25-year follow-up after lung transplantation at Lund University Hospital in Sweden: superior results obtained for patients with cystic fibrosis. Interact CardioVasc Thorac Surg. 2016; 23: 65-73.

9 Gutierreza C, Al-Faifib S, Chaparroc C, et al. The effect of recipient's age on lung transplant outcome. Am J Transplant. 2007; 7: 1271-1277.

10 Thabut G, Ravaud P, Christie JD, et al. Determinants of the survival benefit of lung transplantation in patients with chronic obstructive pulmonary disease. Am J Respir Crit Care Med. 2008; 177: 1156-1163.

11 Sikma MA, Hunault CC, van de Graaf EA, et al. High tacrolimus blood concentrations early after lung transplantation and the risk of kidney injury. Eur J Clin Pharmacol. 2017; 73: 573-580.

12 Meyer KC, Nunley DR, Dauber JH, et al. Neutrophils, unopposed neutrophil elastase, and alpha1-antiprotease defenses following human lung transplantation. Am J Respir Crit Care Med. 2001; 164: 97-102.

Copyright @ERS 2017 